\title{
Educação e desigualdades
}

na pandemia da COVID-19:

realidade e desafios para

\section{as políticas públicas brasileiras}

\author{
Marcio Santos da Natividade
}

Jane Mary de Medeiros Guimarães ${ }^{2}$

Samilly Silva Miranda ${ }^{3}$

Osvaldo Barreto Filho ${ }^{4}$

Erika Santos de Aragão ${ }^{5}$

1 Professor adjunto do Instituto de Saúde Coletiva (ISC) da Universidade Federal da Bahia (UFBA), doutorado em Saúde Pública com ênfase em Epidemiologia pelo ISC da UFBA.

2 Professora adjunta da Universidade Federal do Sul da Bahia (UFSB), doutorado em Saúde Pública pelo Instituto de Saúde Coletiva (ISC) da Universidade Federal da Bahia (UFBA).

3 Professora adjunta do Instituto de Saúde Coletiva (ISC) da Universidade Federal da Bahia (UFBA), doutorado em Saúde Coletiva pela Universidade Estadual de Feira de Santana (UEFS).

4 Professor aposentado da Escola de Administração da Universidade Federal da Bahia (UFBA), mestrado em Administração pela UFBA.

5 Professora adjunta do Instituto de Saúde Coletiva (ISC) da Universidade Federal da Bahia (UFBA), doutorado em Saúde Pública pelo ISC da UFBA.

NATIVIDADE, M. S. da; GUIMARÃES, J. M. de M; MIRANDA, S. S.; BARRETO FILHO, O. ; ARAGÃO, E. S. de. Educação e desigualdades na pandemia da COVID-19: realidade e desafios para as políticas públicas brasileira. In: BARRETO, M. L.; PINTO JUNIOR, E. P.; ARAGÃO, E.; BARRAL-NETTO, M. (org.). Construção de conhecimento no curso da pandemia de COVID-19: aspectos biomédicos, clínico-assistenciais, epidemiológicos e sociais. Salvador: Edufba, 2020. v. 2. DOI: https://doi.org/10.9771/9786556300757.027 


\section{Introdução}

Uma das discussões mais controversas da atualidade é a respeito da reabertura das unidades escolares com a retomada das aulas presenciais. Como em outras inquirições da pandemia por COVID-19, até o presente momento não existem no país pesquisas consolidadas e irrefutáveis sobre quais seriam os prováveis riscos da convivência escolar para alunos, professores e suas respectivas famílias. Então, será que os riscos assumidos com o retorno das aulas são maiores do que a perda de aprendizagem? Na falta de certezas, qual seria o bom senso?

Refletir sobre a educação em tempos de pandemia é considerar tanto os riscos inerentes às atividades de ensino presenciais, quanto a sua importância para o desenvolvimento cognitivo e crescimento do aluno.

"A escola presencial é polifônica. Os sons se espalham pelos ambientes e dão sentido ao espaço educativo. O espaço da escola é mágico. Nele se realiza o milagre permanente do aprender e de abrir-se para o mundo". (KENSKI, 2003, p. 53-54) 
A pandemia da COVID-19 vem exigindo dos gestores públicos de todos os países acometidos a reagirem de forma rápida e oportuna para o controle da disseminação da doença. A principal estratégia adotada para minimizar os impactos dessa enfermidade tem sido o distanciamento social, que inclui, entre outros, o fechamento provisório de escolas e instituições de ensino superior. Essa medida trouxe desafios ao setor educacional, sobretudo em relação ao aprofundamento das desigualdades, bem como da visibilidade das mesmas, principalmente quanto às diferenças em relação ao acesso às tecnologias interativas, rede de internet, infraestrutura das escolas públicas, além da exposição aos riscos de transmissão e de adoecimento dos discentes e docentes.

Muito se tem discutido sobre as possibilidades de retorno das atividades de ensino, e uma das questões levantadas refere-se à utilização da Educação a Distância (EAD), através do uso de Ambientes Virtuais de Aprendizagem (AVA) ou tecnologias interativas - síncronas ou assíncronas - ou ensino híbrido - combinação do ensino presencial com o ensino on-line. Entende-se, no entanto, que não se trata apenas da mudança na modalidade de ensino. O sucesso dessa estratégia requer a superação dos desafios, ou seja, transformar realidades que perpassam pelas estruturas físicas deficitárias das unidades escolares da rede pública de ensino, investir na formação de professores e estudantes para o uso adequado das Tecnologias da Informação e Comunicação (TICs), disponibilizar o acesso a computadores e rede de internet para docentes e discentes. Ou seja, refletir sobre as especificidades econômicas, culturais, sociais dos atores envolvidos nesse processo.

Nesse sentido, considerando a importância de fortalecer a agenda de discussão do impacto da pandemia na educação brasileira, este capítulo propõe discutir os aspectos relacionados às desigualdades sociais no contexto educacional, os desafios atuais 
e futuros, e as possíveis estratégias que possam minimizar os danos causados no processo de aprendizagem e ensino.

\section{Desigualdade e educação no contexto da pandemia}

Do ponto de vista econômico, à medida que a COVID-19 avança pelo mundo já se delineia uma recessão global com a aceleração do desemprego e queda de renda média da população. Para os próximos anos, as projeções realizadas pelo Banco Mundial, pelo Fundo Monetário Internacional (FMI) e pela Comissão Econômica para a América Latina e o Caribe (Cepal) para América Latina, especialmente para o Brasil, prevê uma provável retração econômica em 2020 e, consequentemente, em 2021, com a lenta retomada do crescimento econômico, agravando ainda mais as condições de vida da população mais vulnerável, segundo o Banco Mundial. Apesar dos impactos na economia decorrentes da pandemia, os investimentos em educação devem e/ou deveriam ser mantidos e ampliados. (DIAS; PINTO, 2020) A afirmação da Organização das Nações Unidas para a Educação, a Ciência e a Cultura (Unesco), quanto a queda na aprendizagem aponta que esta poderá alastrar-se por mais de uma década se não forem criadas políticas públicas que invistam em melhorias de infraestrutura, tecnologias, formação, metodologias e salários, além do reforço da alimentação escolar, melhor aproveitamento do tempo, tutoria fora do horário usual das aulas e material adicional, quando possível. (UNESCO, 2020)

Em todo o mundo, estima-se que mais de um bilhão de escolares estejam fora das escolas. (UNESCO, 2020; WANG et al., 2020) Apesar do efeito positivo do fechamento de escolas na redução no pico da doença e na taxa de infecção (MILNE; XIE, 2020; 
SJÖDIN et al., 2020), essa estratégia trará impactos significativos principalmente aos estudantes que vivem em situação de vulnerabilidade, nas suas condições de vida e de saúde, como acontece com grande parte dos estudantes brasileiros. Ou seja, a descontinuidade do aprendizado aumenta as desigualdades, afetando de forma desproporcional os estudantes com condição de vida mais precária. (ECDC, 2020)

No Brasil, o último Censo Escolar da Educação Básica revela que em 2019 foram matriculados cerca de 47,8 milhões de estudantes nas 180,6 mil escolas de educação básica. As escolas públicas responderam por cerca de 38,7 milhões, ou seja, 80,9\% do total de matriculados, concentrados principalmente na área urbana (88,7\%). A desigualdade se expressa quando se identifica que apenas $63,4 \%$ dessas escolas públicas possuem acesso à internet, enquanto nas escolas particulares a proporção é de 96\%. (SILVA; ZIVIANI; GHEZZ, 2019) Nesse contexto, a internet é a principal ferramenta para que a escola reconecte o seu estudante, possibilitando a orientação técnica, pedagógica e psicológica para o acesso ao conhecimento, informação, conteúdos culturais, diversão e lazer, mitigando as repercussões do isolamento social. Evidencia-se uma perversidade da situação para grande parte dos estudantes, principalmente aqueles da rede pública de ensino, que fica de fora desse circuito por não dispor de condições adequadas de conectividade para interagir com o ensino on-line.

No plano pedagógico, a principal medida adotada pelas redes de educação foi o ensino remoto, mas não existem informações concretas sobre como esse ensino se desenvolveu nas 5.570 redes municipais, nas 26 redes estaduais e na rede do Distrito Federal, não se sabe quais os impactos dessa forma de ensino sobre o processo de aprendizagem dos estudantes. O Ministério da Educação (MEC) praticamente se omitiu de formular qualquer política pública de apoio às escolas de estados e municípios para 
a implementação do ensino remoto. O MEC também não adotou nenhum mecanismo de coordenação e de acompanhamento das redes municipais e estaduais de educação para aferir minimamente como estavam se desenvolvendo as atividades através do ensino remoto. Não se tem um quadro do que efetivamente ocorreu, não se sabe qual a efetividade dessa modalidade de ensino no processo de aprendizagem dos estudantes, principalmente para os das escolas públicas.

Segundo Silva, Ziviani e Ghezz (2019), no Brasil, a internet é a reprodução da desigualdade no mundo real. Alardeada como uma ferramenta totalmente democrática, a internet está cada vez mais popular em todo o globo. Em nosso país, no ano de 2017, apenas cerca de $61 \%$ dos domicílios tinham acesso à internet. Os dados apresentados revelam as desigualdades de classes: $90 \%$ das pessoas das classes " $A$ " e " $B$ " têm acesso à internet; enquanto apenas $42 \%$ das classes " $D$ ” e "E" estão conectadas. Mais de $70 \%$ dos usuários encontram-se nas áreas urbanas. Os usuários mais frequentes são aqueles que têm maior renda, escolaridade, entre outras características que definem os padrões socioeconômicos das classes "A" e "B”. (SILVA; ZIVIANI; GHEZZ, 2019) Esses dados revelam a existência de uma estrutura de reprodução de exclusão e desigualdade que são materializadas no acesso à internet e no uso das tecnologias de informação, portanto distante de ser universal e igualitário como se apregoa.

Nesse cenário de incerteza, é importante identificar quais políticas podem maximizar a eficácia do ensino remoto no processo de aprendizagem. O Estado, as escolas e as famílias precisam se mobilizar para não desencadear uma histerese da crise da COVID-19 na educação. (OECD, 2020) De um lado, é preciso considerar o ensino on-line como uma ferramenta importante para sustentar o desenvolvimento de habilidades durante o fechamento das escolas, mas que requer acesso universal à infraestrutura 
- computadores e rede de internet - e preparação adequada entre professores e estudantes para o ensino e a aprendizagem on-line; por outro, ao considerarmos as dificuldades para a garantia de acesso aos recursos essenciais para o ensino on-line, bem como a impossibilidade dos cursos em que atividades práticas laboratoriais ou extramuros são essenciais para a formação, é crucial planejar estratégias para o retorno gradual do ensino presencial, combinado com o ensino remoto. Assim, são discutidos a seguir alguns desafios e possíveis medidas a serem considerados em todos os níveis de ensino para o possível retorno das atividades presenciais.

\section{Os principais prováveis riscos do coronavírus na educação}

A complexidade para organizar o retorno às aulas, considerando as 5.570 redes municipais, as 26 redes estaduais e a rede do Distrito Federal, inclui as reflexões referente tanto ao risco de contaminação dos envolvidos direta ou indiretamente nesse processo - alunos, professores, funcionários e seus familiares -, quanto o impacto no aprendizado, no desenvolvimento mental e social dos alunos e na ampliação das desigualdades na educação e nas perspectivas de vida a longo prazo.

Considerando os riscos de contaminação, para a educação infantil e níveis fundamental e médio, é preciso ponderar que crianças e adolescentes de todas as idades são suscetíveis à infecção por SARS-CoV-2. Embora representem em média de $1 \%$ a $5 \%$ dos casos diagnosticados (LUDVIGSSON, 2020), sendo a maioria com manifestações clínicas leves e bom prognóstico, aquelas com doenças subjacentes - como doenças cardíacas, pulmonares e das vias aéreas congênitas, doenças renais e cardíacas 
crônicas, desnutrição, doenças metabólicas hereditárias, doenças da imunodeficiência, tumor etc. - provavelmente se tornarão casos graves. (GÖTZINGER et al., 2020; SHEN et al., 2020) Nesse caso, faz-se necessário a triagem prévia quanto às condições sistêmicas das crianças e adolescentes, quando for oportuno o retorno das atividades de ensino presenciais, além de seguir protocolos de biossegurança previamente estabelecidos para o controle da disseminação.

A literatura traz discussões importantes sobre a capacidade das crianças infectadas de disseminar o SARS-CoV-2. Os resultados apontam uma variação entre 5 a 10\% de crianças serem o caso-índice nas famílias. (JING et al., 2020; ZHU et al., 2020) Em um estudo escolar de New South Wales, Austrália, não foi encontrada evidência de crianças infectando professores. (NATIONAL CENTRE FOR IMMUNISATION AND SURVEILLANCE, 2020) Embora esses achados favoreçam a possibilidade de retorno às aulas para crianças, é preciso considerar os resultados com cautela, sempre levando em consideração a condição epidemiológica da região. Mesmo que as crianças tenham uma baixa capacidade de transmissão, o pouco que se transmite pode favorecer a ocorrência de novos surtos da COVID-19.

Para o ensino superior, segundo os dados do censo da educação superior, a idade média dos alunos matriculados em cursos presenciais é de 26 anos, indicando uma faixa etária de adultos jovens. (INEP, 2015) De acordo com o boletim do Ministério da Saúde da Semana Epidemiológica 52 (21 de fevereiro a 27 de fevereiro de 2021 -, apenas 3\% das hospitalizações por Síndrome Respiratória Aguda Grave (SARS) foram de indivíduos entre 20 a 29 anos. (BOLETIM EPIDEMIOLÓGICO ESPECIAL, 2021) Na Bahia, essa mesma faixa etária corresponde a 17,73\% dos casos confirmados, e representa 1,3\% dos óbitos de 2020 a 2021. (BOLETIM EPIDEMIOLÓGICO COVID-19, 2021) Apesar de essas faixas etárias 
serem consideradas de baixo risco, estas apresentam potencial de transmissão para toda a comunidade escolar, incluindo docentes, discentes, técnico-administrativos, prestadores de serviços, colaboradores e fornecedores de materiais e insumos, o que requer vigilância constante de toda a universidade de modo a prevenir, conter e mitigar a propagação do vírus.

Quanto aos professores, a média de idade na educação básica é de 41 anos, 39 anos para ensino médio e 38 anos para educação superior. (CARVALHO, 2018; INEP, 2018) Além do fator idade, os docentes podem pertencer ao grupo de risco por apresentar alguma comorbidade (obesidade, diabetes, hipertensão, por exemplo); ou morar com alguém que pertence ao grupo de risco, dentre outras questões. Assim, independentemente das medidas de prevenção implementadas para crianças e adolescentes, a proteção de professores, educadores e cuidadores é fundamental, portanto, para o retorno às aulas com segurança de todos os indivíduos envolvidos, protocolos vêm sendo planejados por diversas frentes, considerando inclusive a importância da vigilância prospectiva e cuidadosa. (WALGER, 2020)

\section{Protocolos para o retorno às aulas presenciais}

O MEC publicou Protocolo de Biossegurança para Retorno das Atividades nas Instituições Federais de Ensino, mas considera relevante que as Instituições de Ensino constituam comissão local para definição e adoção de protocolos próprios. Qualquer retorno deve ser baseado nas informações epidemiológicas de cada território e nas orientações das autoridades sanitárias competentes. Foram consideradas tanto medidas coletivas, quanto individuais (Quadro 1). 
Quadro 1 - Medidas coletivas e individuais a serem adotadas para o retorno gradual das atividades de ensino superior

\begin{tabular}{|c|c|}
\hline \multicolumn{1}{|c|}{ Medidas Coletivas } & Medidas Individuais \\
\hline $\begin{array}{c}\text { Realizar trabalho escalonado entre equipes } \\
\text { e com distanciamento social. }\end{array}$ & $\begin{array}{c}\text { Utilizar máscaras, higienizar as mãos e } \\
\text { utilizar regras de etiqueta respiratória. }\end{array}$ \\
\hline $\begin{array}{c}\text { Garantir ventilação do ambiente - abertura } \\
\text { de portas e janelas. }\end{array}$ & Evitar cumprimentos próximos. \\
\hline $\begin{array}{c}\text { Organizar a rotina de limpeza do ambiente } \\
\text { de trabalho e equipamentos. }\end{array}$ & Manter distância de 1,5m entre as pessoas. \\
\hline $\begin{array}{c}\text { Possibilitar atividades laborais e de ensino } \\
\text { remoto para colaboradores, docentes e } \\
\text { discentes do grupo de risco. }\end{array}$ & Manter cabelo preso e limitar o uso de \\
\hline $\begin{array}{c}\text { Realizar reuniões e eventos à distância com } \\
\text { uso de tecnologias virtuais. }\end{array}$ & Não compartilhar objetos pessoais. \\
\hline
\end{tabular}

Fonte: Brasil (2020).

Através do Guia de Implementação de Protocolos de Retorno das Atividades Presenciais nas Escolas de Educação Básica, o MEC apresenta as normas técnicas de segurança em saúde, recomendações de ações sociais e pedagógicas, para o retorno às aulas presenciais nas escolas de educação básica, apesar dessa decisão caber aos estados e municípios. Orienta o uso obrigatório de máscaras, a garantia de um distanciamento mínimo de um metro, o uso de Equipamentos de Proteção Individual (EPIs) para os profissionais de ensino e a adoção de regimes de revezamento de equipes. Dentre as medidas pedagógicas, recomenda o uso do ensino híbrido, a realização de avaliações diagnósticas e formativas para orientar programas de recuperação da aprendizagem. Sugere também a criação do Conselho Escolar Ampliado para coordenar o processo de retomada das aulas, que tem entre outras funções, buscar parcerias locais que possam contribuir com a 
biossegurança do ambiente escolar, o acompanhamento do monitoramento e triagem de alunos, professores e funcionários.

Em decorrência das diferenças entre as incidências e coeficientes de mortalidade em todo o mundo, os países estão em diferentes estágios em relação a como e quando planejam reabrir as escolas. As decisões frequentemente são tomadas pelo governo federal ou estadual, no entanto, as autoridades locais sempre devem compor a tomada de decisão, levando em conta o princípio da beneficência. Logo, o governo, independente da instância, deverá garantir a segurança dos estudantes, funcionários, professores e suas famílias. (UNESCO et al., 2020)

A Organização Mundial da Saúde (OMS), a Unesco e o Banco Mundial também publicaram diretrizes sobre a reabertura de escolas. Para tanto, algumas dimensões-chave relacionadas à política, financiamento, operações seguras, aprendizado, alcance dos marginalizados e bem-estar/proteção, devem ser consideradas. A resposta deve favorecer a melhoria da aprendizagem, aumentar acesso equitativo à educação e fortalecer a proteção, a saúde e a segurança dos estudantes. O governo precisa, portanto, estabelecer metas a serem cumpridas, antes, após e durante a reabertura: 
Quadro 2 - Adaptação das estratégias para o retorno gradual das aulas presenciais propostas pela Unesco

\begin{tabular}{|c|c|}
\hline \multicolumn{2}{|r|}{ Estratégias para o retorno gradual das aulas presenciais } \\
\hline Antes & $\begin{array}{c}\text { Fornecer orientações nacionais sobre a abertura de forma segura; Criar } \\
\text { protocolos sobre medidas físicas de distanciamento e de higiene; Criar } \\
\text { políticas de proteção para grupos de risco - alunos, funcionários e } \\
\text { professores; Desenvolver estratégias de financiamento para melhorias na } \\
\text { infraestrutura das escolas/universidades, que garantam condições sanitárias; } \\
\text { Fornecer aos professores e líderes escolares suporte e treinamento em } \\
\text { aprendizado remoto. }\end{array}$ \\
\hline Durante & $\begin{array}{l}\text { Fortalecer o diálogo entre comunidade escolar, pais e filhos; Garantir que } \\
\text { as escolas tenham acesso à água potável, a estações de lavagem de mãos, } \\
\text { a material de limpeza, dentre outros; Equipar e treinar as equipes de } \\
\text { limpeza quanto à desinfecção de ambientes; Monitorar a saúde dos alunos, } \\
\text { professores e funcionários; Promover práticas de higiene em todos os níveis } \\
\text { e para todo o pessoal do sistema escolar, criando rotinas de higienização; } \\
\text { Mitigar a exacerbação da desigualdade; Ampliar os serviços de apoio } \\
\text { psicossocial; Escalonar o início e o fim do dia escolar, com possibilidades } \\
\text { de alternância entre aulas presenciais e a distância; Limitar o número de } \\
\text { alunos de acordo com o espaço da sala de aula - mínimo de um metro por } \\
\text { estudante; Uso de máscaras obrigatório, até que se considere o controle } \\
\text { efetivo da doença; Reorganizar a entrega da merenda escolar e/ou os } \\
\text { espaços de venda de alimentos. }\end{array}$ \\
\hline Após & $\begin{array}{l}\text { Desenvolver modelos de decisão em casos de ressurgimento da transmissão } \\
\text { comunitária; Garantir que as informações sobre higiene estejam amplamente } \\
\text { disponíveis e acessíveis; Em caso de adaptação da carga horária, aumentar } \\
\text { os investimentos em aprendizado remoto; Avaliar o desempenho dos alunos; } \\
\text { Compartilhar informações seguras e acessíveis, inclusive para pessoas com } \\
\text { deficiência auditiva ou visual que estimulem o autocuidado e reduzam o } \\
\text { medo e a insegurança entre alunos, professores, funcionários e familiares. }\end{array}$ \\
\hline
\end{tabular}

Fonte: adaptado da Unesco e demais autores (2020).

Embora os protocolos sinalizem a importância do planejamento e adequação das medidas de biossegurança para a reabertura 
de escolas e universidades, é fundamental que a infraestrutura de cada espaço escolar seja adaptada à nova realidade. Infelizmente, as escolas brasileiras possuem condições distintas de infraestrutura, recursos financeiros, adequação de força de trabalho, interlocução com o sistema de saúde, que dificulta a capacidade de atender todas às orientações supracitadas. (PEREIRA et al., 2020) Essas desigualdades podem ser percebidas quando se avalia, por exemplo, a frequência de acesso a rede pública de esgoto que, segundo informações do Censo Escolar 2019, apenas 44\% das escolas brasileiras a possuem, com piores condições nas regiões Norte e Nordeste. (KUBOTA, 2020) Nesse sentido, os investimentos necessários para o retorno das atividades de ensino irão requerer uma avaliação criteriosa, de modo que estes sejam realizados de forma equânime, com o intento de minimizar as iniquidades, que já são tão marcantes no nosso sistema educacional.

\section{A modalidade EAD seria a solução mais viável para o retorno às aulas pós-pandemia?}

A Portaria $n^{\circ} 343$, de 17 de março de 2020 e a Medida Provisória ${ }^{\circ}$ 934, de $1^{\circ}$ de abril de 2020 , permitiram, em caráter excepcional, a substituição de aulas presenciais por aulas remotas que utilizam meios e tecnologias de informação e comunicação, nas instituições de educação superior no âmbito federal de ensino. Seguindo essas orientações, diversas Instituições de Ensino Superior aderiram à modalidade EAD como forma de dar continuidade ao ano letivo. (CASTAMAN; RODRIGUES, 2020)

A mudança do ensino para a modalidade EAD no formato on-line perpassa diversas questões; no Brasil, destaca-se a dificuldade de acesso dos alunos. A transposição das aulas presenciais para on-line pode não resultar em experiências positivas, 
principalmente para os estudantes que usarão smartphones, pois há diferenças significativas relacionadas ao tamanho da fonte, ao áudio, à digitalização das atividades, dentre outras questões. (REIMERS, 2020)

Os desafios da modalidade EAD se apresentam de múltiplas formas. Alguns docentes reconhecem que o excesso de atividades desestimula os processos de ensino e aprendizagem, bem como a existência de iniciativas interessantes como a associação de diferentes disciplinas, em decorrência dos limites de conexão, culmina numa forma de superar o cartesianismo. (CASTAMAN; RODRIGUES, 2020) No entanto, há de se considerar, no caso em que as atividades realizadas na modalidade a distância utilizaram metodologias, conteúdos e atividades educacionais adequadas, além da ampla acessibilidade, o ensino remoto resolveu os problemas intertemporais durante e após a epidemia, essa modalidade pode se fortalecer no longo prazo. (SENHORAS, 2020)

Nas escolas de ensino básico e fundamental, as férias foram antecipadas, algumas unidades escolares paralisaram outras continuaram através do ensino na modalidade EAD, impactando nos professores e famílias, uma vez que a educação domiciliar trouxe mudanças para o aprendizado, sobrecarregando as famílias no acompanhamento. (BURGESS; SIEVERTSEN, 2020)

Outros impactos negativos podem ser manifestados pelo aumento da evasão escolar, ações estratégicas emergenciais para a eventual continuidade dos estudos, além do esforço no planejamento para a normalização dos ciclos escolares no médio prazo. (SENHORAS, 2020) Os dados do INEP (2018) revelam que apesar do número de vagas ofertadas na modalidade $\mathrm{EAD}$ ter superado a presencial no Brasil, a evasão também é maior nessa modalidade, justificada pela falta de apoio acadêmico, problemas com a tecnologia e falta de apoio administrativo. (DEMO, 2002) 
Nesse contexto, a modalidade EAD não pode ser vista como a solução dos problemas enfrentados pela educação no mundo e no Brasil. A mudança repentina para o uso da instrução digital pode levar a resultados abaixo do ideal se comparada com o ensino presencial, já que professores, alunos e escolas tiveram que se ajustar inesperadamente a uma situação nova. Ainda se tem a problemática do acesso a computadores e conexão de banda larga em casa; da dificuldade em relação à carga horária de ensino e da quantidade de conteúdos a serem ofertados; da falta de habilidades digitais básicas entre alunos e professores, dificultando a adaptação à nova situação. (OCDE, 2020)

Infelizmente, é dentro desse ambiente confuso, mais de um ano depois do fechamento das escolas, num quadro em que o número diário de novos contaminados e de novas mortes ainda é bastante elevado e instável, que se discute a reabertura das escolas. Reina a mais absoluta confusão. Pesquisas de opinião indicam que mais de $70 \%$ da população acha que as escolas não devem reabrir, o que reflete, em certa medida, como a educação é pouco valorizada em nossa sociedade, há um descaso em relação à educação. No Brasil, muitas atividades, após muita pressão, já retornaram seu ritmo normal: construção civil, comércio, bares e restaurantes, centros comerciais, atividades de lazer, praias. O cenário descrito revela a urgência de adoção de políticas públicas e de mecanismos de coordenação principalmente do MEC no âmbito federal para minimizar os impactos da pandemia sobre o setor educacional. A persistir o descaso com a educação, certamente acarretará impactos negativos de longo prazo para a comunidade escolar - estudantes e professores -, aos seus familiares e, consequentemente, para o desenvolvimento do país. 


\section{Considerações finais}

O retorno das atividades não significa o relaxamento do risco de adoecimento pela COVID-19, por isso, é imprescindível o planejamento desse retorno com cautela, garantindo a manutenção de vigilância e o monitoramento de risco. Afinal, enquanto houver casos de indivíduos com diagnóstico positivo, há risco de adoecimento e novos surtos, conforme evidenciam as experiências em outros países. Ao mesmo tempo, não se pode esquecer os diversos prejuízos a longo prazo para o alunado, como aqueles matriculados no $3^{\circ}$ ano do ensino médio, grupo que se prepara para o acesso à educação superior, a exemplo do Exame Nacional do Ensino Médio (ENEM); crianças em idade pré-escolar; $1^{\circ}$ e $2^{\circ}$ ano do ensino fundamental - fase da alfabetização; estudantes em transição da fase I para a fase II do ensino fundamental; e estudantes em transição para o ensino médio.

Admitindo-se o retorno às aulas, deve-se considerar a necessidade de um processo de avaliação dos estudantes que permita verificar o nível de aprendizagem com que cada estudante está retornando à escola, ou seja, medir o grau de efetividade das atividades remotas; organizar o processo de aprendizagem considerando as diferenças de nível de aprendizagem do estudante; considerar os protocolos pedagógicos que serão utilizados e os de convivência social entre a comunidade escolar, deve-se adequar a estrutura física das escolas seguindo parâmetros descritos nos protocolos supracitados.

Em caso de impossibilidade de retorno, é imperioso otimizar a utilização das tecnologias, visto que estas só fazem sentido quando se busca caminhos alternativos de renovação educacional e transformação do sujeito e, portanto, da sociedade em que vivemos. Essa estratégia não pode ser mais uma ferramenta que acentue as iniquidades sociais, tão marcantes em nosso país. 


\section{Referências}

BOLETIM EPIDEMIOLÓGICO COVID-19. Salvador: Secretaria da Saúde, n. 405, 3 maio 2021. Disponível em: http://www.saude.ba.gov.br/wp-content/ uploads/2021/05/BOLETIM_ELETRONICO_BAHIAN_405_03052021.pdf. Acesso em: 4 maio 2021.

BOLETIM EPIDEMIOLÓGICO ESPECIAL: doença pelo coronavírus - COVID-19. Brasília, DF: Ministério da Saúde, n. 52, 2021. Disponível em: https://www.gov.br/ saude/pt-br/media/pdf/2021/marco/05/boletim_epidemiologico_covid_52_final2. pdf. Acesso em: 4 maio 2021.

BRASIL. Ministério da Educação. Censo da educação superior 2015. Brasília, DF, 2015. Disponível em: http://portal.mec.gov.br/component/tags/tag/32044-censoda-educacao-superior. Acesso em: 9 jul. 2020.

BRASIL. Ministério da Educação. Secretário de Educação Superior. Secretário de Educação Profissional e Tecnológica. Protocolo de biossegurança para retorno das atividades nas instituições federais de ensino. Brasília, DF, 2020. Disponível em: https://vps3574.publiccloud.com.br/cartilhabio.pdf. Acesso em: 10 jul. 2020.

BURGESS, S.; SIEVERTSEN, H. H. Schools, skills, and learning: The impact of COVID-19 on education. VoxEU, [s. I.], 1 abr. 2020. Disponível em: https://voxeu. org/article/impact-COVID-19-education. Acesso em: 14 maio 2020.

CASTAMAN, A. S.; RODRIGUES, R. A. Educação a distância na crise COVID-19: um relato de experiência. Research, Society and Development, Vargem Grande Paulista, SP, v. 9, n. 6, p. e180963699, 2020.

CARVALHO, M. R. V. de. Perfil do professor da educação básica. Brasília, DF: INEP, 2018. (Série Documental. Relatos de Pesquisa, n. 41). Disponível em: http://portal. inep.gov.br/informacao-da-publicacao/-/asset_publisher/6JYIsGMAMkW1/ document/id/1473981. Acesso em: 7 out. 2010.

DEMO, P. Complexidade e aprendizagem: a dinâmica não-linear do conhecimento. São Paulo: Atlas, 2002.

DIAS, E.; PINTO, F. A educação e a COVID-19. Ensaio: avaliação e políticas públicas em educação, Rio de Janeiro, v. 28, n.108, p. 545-554, 2020. 


\section{EUROPEAN CENTRE FOR DISEASE PREVENTION AND CONTROL - ECDC.}

Rapid risk assessment: outbreak of novel coronavirus disease 2019 (COVID19): increased transmission globally-fifth update. 2 Mar. 2020. Disponível em: https://www.ecdc.europa.eu/sites/default/files/documents/RRA-outbreaknovel-coronavir-us-disease-2019-increase-transmission-globally-COVID-19.pdf. Acesso em: 7 abr. 2020.

GÖTZINGER, F. et al. COVID-19 in children and adolescents in Europe: a multinational, multicentre cohort study. The Lancet. Child \& Adolescent Health, Cambridge, v. 4, n. 9, p. 653-661, 2020.

INEP. Censo da educação superior 2018: notas estatísticas. Brasília, DF, 2018. Disponível em: http://download.inep.gov.br/educacao_superior/censo_superior/ documentos/2019/censo_da_educacao_superior_2018-notas_estatisticas.pdf. Acesso em: 7 out. 2020.

INEP. Resultados do censo escolar 2019. Brasília, DF, 2019. Disponível em: http:// portal.inep.gov.br/web/guest/resultados-e-resumos. Acesso em: 4 abr. 2020.

JING, Q.-L. et al. Household secondary attack rate of COVID-19 and associated determinants. MedRxiv, [s. I.], 2020. DOI: 10.1101/2020.04.11.20056010.

Disponível em: https://www.medrxiv.org/content/10.1101/2020.04.11.20056010v1. Acesso em: 7 out. 2020.

KUBOTA, L. C. A infraestrutura sanitária e tecnológica das escolas e a retomada das aulas em tempos de COVID-19. Brasília, DF: IPEA, 2020. (Nota Técnica, n. 70). Disponível em: https://www.ipea.gov.br/portal/images/stories/PDFs/nota tecnica/200715_nt_diset_n_70_web.pdf. Acesso em: 9 out. 2020.

KENSKI, V. M. Tecnologias e ensino presencial e a distância. Campinas: Papirus, 2003.

LUDVIGSSON, J. F. Systematic review of COVID-19 in children shows milder cases and a better prognosis than adults. Acta Paediatrica, Oslo, v. 109, n. 6, p. 1088-1095, 2020.

MILNE, G. J.; XIE, S. The effectiveness of social distancing in mitigating COVID-19 spread: a modelling analysis. MedRxiv, [s. I.], 2020. DOI: https://doi.org/1 0.1101/2020.03.20.20040055. Disponível em: https://www.medrxiv.org/ content/10.1101/2020.03.20.20040055v1. Acesso em: 13 out. 2020.

NATIONAL CENTRE FOR IMMUNISATION AND SURVEILLANCE. COVID-19 in schools - the experience in NSW. 2020. Disponivel em: http://ncirs.org.au/sites/ default/files/2020-04/NCIRS\%20NSW\%20Schools\%20COVID_Summary_FINAL\%20 public_26\%20April\%202020.pdf. Acesso em: 10 jul. 2020. 
ORGANISATION FOR ECONOMIC CO-OPERATION AND DEVELOPMENT - OECD. Education and COVID-19: Focusing on the long-term impact of school closures. 29 June 2020. Disponível em: http://www.oecd.org/coronavirus/policy-responses/ education-and-COVID-19-focusing-on-the-long-term-impact-of-school-closures2cea926e/. Acesso em: 7 out. 2010.

PEREIRA, I. D. F. et al. Manual sobre biossegurança para reabertura de escolas no contexto da COVID-19. Rio de Janeiro: EPSJV, 2020. Disponível em: https:// portal.fiocruz.br/sites/portal.fiocruz.br/files/documentos/manual_reabertura.pdf. Acesso em: 13 out. 2020.

REIMERS, F. M. Um roteiro para guiar a resposta educacional à Pandemia da COVID-19 de 2020. [s. I.], 30 mar. 2020. Disponível em: https://globaled.gse.harvard. edu/files/geii/files/um_roteiro_para_guiar_a_resposta_educacional_a_pandemia_da_ COVID-19_reimersschleicher_ceipe_30032020_1.pdf. Acesso em: 7 out. 2020.

SENHORAS, E. M. Coronavírus e educação: análise dos impactos assimétricos. Boletim de Conjuntura, Boa Vista, RR, v. 2, n. 5, p. 128-136, 2020.

SHEN, K. et al. Diagnosis, treatment, and prevention of 2019 novel coronavirus infection in children: experts' consensus statement. World Journal of Pediatrics, Hangzhou, v. 16, n. 3, p. 223-231, 2020. DOI: 10.1007/s12519-020-00343-7. Disponível em: https://www.ncbi.nlm.nih.gov/pmc/articles/PMC7090771/ Acesso em: 7 out. 2020.

SILVA, F. A. B. da; ZIVIANI, P.; GHEZZ, D. R. As tecnologias digitais e seus usos. Brasília: IPEA, abr. 2019. (Texto para Discussão).

SJÖDIN, J. et al. COVID-19 health care demand and mortality in Sweden in response to non pharmaceutical (NPIs) mitigation and suppression scenarios. MedRxiv, [s. I.], 2020. DOI: https://doi.org/10.1101/2020.03.20.20039594.

Disponível em: https://www.medrxiv.org/content/10.1101/2020.03.20.20039594v3. Acesso em: 14 maio 2020.

UNESCO. Building peace in the minds of men and women. COVID-19 Educational Disruption and Response. 2020. Disponível em: https://en.unesco.org/covid19/ educationresponse. Acesso em: 4 abr. 2020.

UNESCO et al. Framework for reopening schools. June 2020.

Disponível em: https://www.unicef.org/documents/framework-reopening-schools. Acesso em: 10 jul. 2020. 
WALGER, P. et al. Children and adolescents in the COVID-19 pandemic: Schools and daycare centers are to be opened again without restrictions; the protection of teachers, educators, carers and parents and the general hygiene rules do not conflict with this. GMS Hygiene and Infection Control, Berlin, 2020.

WANG, G. et al. Mitigate the effects of home confinement on children during the COVID-19 outbreak. Lancet, London, v. 395, n. 10228, p. 945-947, 2020.

ZHU, Y. et al. Children are unlikely to have been the primary source of household SARS-CoV-2 infections. MedRxiv, [s. I.], 2020. Disponível em: https://www.medrxiv. org/content/10.1101/2020.03.26.20044826v1. Acesso em: 7 out. 2020. 